\title{
Editorial
}

\section{Biventricular pacing in patients with severe heart failure: has the time come?}

In recent years there has been a growing interest in using cardiac pacing as additive treatment in severe heart failure. It is estimated that $30 \%$ of patients with severe heart failure have intraventricular conduction disturbances mechanically characterised by a discoordinate ventricular contraction pattern and wide QRS complexes. ${ }^{1}$ Multisite biventricular pacing, which restores the synchronisation of the ventricular contraction, could be expected to be beneficial in such patients. A clinical benefit from biventricular pacing in heart failure was first suggested by Bakker and colleagues. $^{2}$ Thereafter acute and short term haemodynamic benefits, including decreases in filling pressures and mitral regurgitation and improvements in diastolic filling and cardiac output, from biventricular pacing were demonstrated. ${ }^{3}{ }^{4}$ It is, however, by no means clear whether acute and short term haemodynamic benefits will translate into long term or indeed into clinical improvements. Moreover, randomised trials are needed to establish the true value of this treatment.

Results from uncontrolled studies suggest that multisite pacing improves selected heart failure patients. ${ }^{5}$ The largest of these is the Insync study. It comprised 68 patients with severe heart failure of mixed aetiology. All patients had severe heart failure, New York Heart Association (NYHA) functional class III-IV, and a QRS duration of at least $150 \mathrm{~ms}$. Significant improvements by biventricular pacing were seen in most patients after three and six months of pacing compared to baseline with regard to NYHA class, six minute walk distance, and quality of life. Moreover, pacing reduced the QRS duration significantly and normalised the intraventricular delay as an indication that ventricular resynchronisation had been achieved.

Hospital care constitutes a major cost in heart failure management. ${ }^{6}$ One recent study demonstrated a reduced need for all cause and heart failure related hospital care in parallel with clinical improvements by biventricular pacing. ${ }^{7}$ Even though pacemaker treatment is not inexpensive, neither is hospital care. If biventricular pacing is proven to be efficient in the long term, it could prove to be cost effective.

Increased sympathetic activity in severe heart failure carries a negative impact on survival. One study indicates that biventricular pacing modifies sympathetic activity in those patients with the highest baseline norepinephrine (noradrenaline) concentrations. ${ }^{8}$ Moreover an increased vagal influence following biventricular pacing and reduced QT dispersion has been observed. If this is true, biventricular pacing could reduce the risk for ventricular arrhythmias.

There are a number of randomised trials on the efficacy and safety of biventricular pacing, the results of which remain to be published. In the PATH CHF (pacing therapy in congestive heart failure) trial, patients with severe heart failure in NYHA III or IV and a QRS duration of at least $120 \mathrm{~ms}$ were included. After an extensive acute invasive evaluation performed during implantation, the patients were randomised to one month each of pacing in the best univentricular mode - that is, right or left ventricular pacing only, no pacing, and biventricular pacing. The study enrolment ended in 1998 and included 54 patients. Interim results indicate a $40 \%$ improvement in the six minute walk distance and a $50 \%$ improvement in quality of life both after one month of the best univentricular mode, in most cases constituted by left ventricular pacing, and one month of biventricular pacing.

The MUSTIC (multisite stimulation in cardiomyopathy) study involves 120 patients. ${ }^{9}$ Enrolment was completed in June 1999. This study includes only NYHA class III heart failure patients with a QRS duration of at least $150 \mathrm{~ms}$. The patients were randomised in a single blind crossover fashion to three months each of biventricular pacing or fixed rate ventricular pacing at a rate of 40 beats per minute. The primary end points are the six minute walk distance and maximal oxygen uptake, with quality of life as a secondary end point. We need the results of these and other ongoing randomised trials to assess whether biventricular pacing can be considered outside the research community.

Presently this treatment should be confined to specialised centres with ongoing research projects in the field. There are several reasons for this: the value of the treatment needs to be firmly established; the left ventricular lead introduction requires training; and pacemaker programming and follow up is more complicated than with conventional pacing. Early and late left ventricular lead dislodgements, cross talk, and left phrenic nerve stimulation, not uncommon in biventricular pacing, are examples of this complexity. Probably, many of these difficulties will be overcome by technical improvements in lead and pacemaker technology.

Biventricular pacing is a promising treatment in patients with severe heart failure with intraventricular conduction disturbances. It helps improve patients by at least one functional class, increases the six minute walk distance by $20-40 \%$, increases the maximal oxygen uptake by $10-40 \%$, and improves quality of life (as assessed by the Minnesota form) by $40-50 \%$. These figures are impressive, even considering the placebo effects of pacemaker implantation, and are comparable or surpass what has been found in drug trials involving similar patients. ${ }^{10}$ From uncontrolled studies it is also clear that not all patients respond to this treatment. Clinical, electrical or echocardiographic predictors of response to pacing are needed in view of the costs involved in pacemaker implantation and follow up. Current knowledge, mainly from the Insync trial, indicates that clinical parameters are poor predictors of response. Neither age, aetiology nor NYHA class are useful to predict a response to pacing. The value of electrical predictors appear equally poor. The currently used electrical predictor-a long QRS duration at baseline-does not appear to suffice. Although a QRS duration of more than $160 \mathrm{~ms}$ indicated an acute haemodynamic response in the PATH CHF trial, ${ }^{4}$ this was not a predictor of a short term clinical response to pacing in the InSync trial. In this study a narrowing of the QRS complex by biventricular pacing was instead found in the responders.

Finally, there are a number of remaining questions. What is the proper stimulation site? How long will short term results last? If there is a long term effect, does biventricular pacing reduce mortality? 
Only the results from rigorously conducted prospective randomised trials can determine the usefulness of biventricular pacing in heart failure. At the present time we need to await these results.

Department of Cardiology,

CECILIA LINDE

Karolinska Hospital,

S-117 76 Stockholm, Sweden

cecilialinde@ks.sc

Supported by the Swedish Heart and Lung Association, and the Swedish Medical Research Council (grant no B96-11626-01)

1 Stevenson WG, Stevenson LW, Middlekauff HR, et al. Improving survival for patients with advanced heart failure: a study of 737 consecutive patients. $\mathcal{F}$ Am Coll Cardiol 1995;26:1417-23.

2 Bakker P, Chin K, Sen A, et al. Biventricular pacing improves functional capacity in patients with end-stage congestive heart failure [abstract]. PACE 1995;18:825.
3 Cazeau S, Ritter P, Lazarus A, et al. Multisite pacing in end-stage heart failure: early experience. PACE 1996;19(PtII): 1748-57.

4 Auricchio A, Stellbrink C, Block M, et al. Effect of pacing chamber and atrioventricular delay on acute systolic function of paced patients with congestive heart failure. Circulation 1999;99:2993-3001.

5 Gras D, Mabo P, Tang T, et al. Multisite pacing as a supplemental treatment of congestive heart failure: preliminary results of the Medtronic Inc InSync of congestive heart failure: preliminary results of the Med

6 McMurray J, Hart W, Rhodes G. An evaluation of the cost of heart failure to the National Health Service in the UK. British Fournal of Medical Economics 1993;6:99-110.

7 Braunschweig F, Linde C, Gadler F, et al. Reduction of hospital days by biventricular pacing. European fournal of Heart Failure 2000. In press.

8 Saxon L, De Marco T, Chatterjee K, et al. Chronic biventricular pacing decreases serum norepinephrine in dilated heart failure patients with the greatest sympathetic activation at baseline [abstract]. PACE 1999;22:830.

9 Daubert C, Linde C, Cazeau S, et al. Protocol design and current status of the European controlled multicentre study on multisite biventricular pacing in severe heart failure: the MUSTIC study. PACE 1999;22:A116.

10 Linde C, Gadler F, Kappenberger L, et al. Does pacemaker implantation carry a placebo effect? Am f Cardiol 1999;83:903-7.

\section{IMAGES IN CARDIOLOGY}

\section{Mycotic pseudoaneurysm of the ascending aorta following aortic valve replacement}

A 71 year old woman presented with lethargy and weakness. One year previously she underwent aortic valve replacement for symptomatic aortic stenosis with a Carpentier-Edwards pericardial valve. Her admission temperature was $101.4^{\circ} \mathrm{F}\left(38.6^{\circ} \mathrm{C}\right)$ and subsequent blood cultures grew methicillin resistant Staphylococcus aureus. Transoesophageal echocardiography (top) revealed an echo free bilobed space (arrow) originating from the ascending aorta approximately $2 \mathrm{~cm}$ above the level of the aortic annulus. The pseudoaneurysm was surrounded by increased echogenicity consistent with inflammation (LA, left atrium). Chest computed tomography (middle) revealed an abscess cavity (arrow) in communication with the proximal ascending aorta ("A"), with contrast extravasation into the abscess cavity surrounded by a large area of mediastinal inflammatory consolidation.

At surgery a large active pseudoaneurysm abscess cavity originating from the old aortotomy suture line was found (bottom). Surgical approach included femoral-femoral cardiopulmonary bypass, with careful repeat sternotomy and dissection of the proximal ascending aorta allowing for cross clamping at the level of the innominate artery take off. The aorta was opened laterally, allowing identification of the origin of the pseudoaneurysm at the medial start site of the previous aortotomy, approximately $2 \mathrm{~cm}$ above the aortic annulus. The abscess cavity contained fresh pus and clotted blood. The abscess and involved aortic wall was debrided, and the ascending aorta was reconstructed by patch aortoplasty using cryopreserved homograft aorta. The bioprosthetic valve did not appear to be involved in the infectious process, and was left in situ.

Following omentoplasty and chest closure the patient was extubated and had an uneventful early postoperative course. Unfortunately she died from complications related to a pulmonary embolus two weeks later.
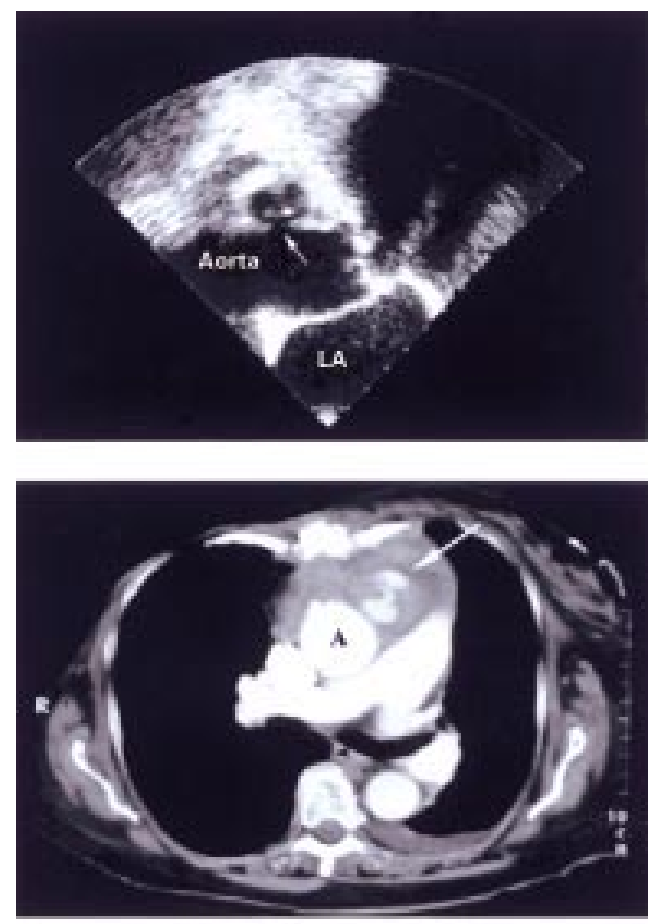

DAVID H ADAMS ALEXANDER KADNER RAYMOND H CHEN

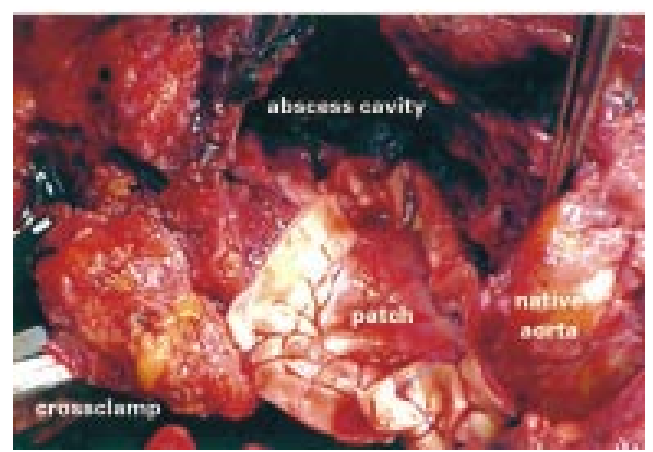

